\title{
Influence of the UV Cure on Advanced Plasma Enhanced Chemical Vapour Deposition Low-k Materials
}

\author{
Patrick Verdonck*, Els Van Besien, Kris Vanstreels, Christos Trompoukis, \\ Adam Urbanowicz ${ }^{\dagger}$, David De Roest ${ }^{1}$, and Mikhail R. Baklanov
}

imec, Kapeldreef 75, 3001 Heverlee, Belgium

${ }^{1}$ ASM, Kapeldreef 75, 3001 Heverlee, Belgium

Received November 12, 2010; accepted December 22, 2010; published online May 20, 2011

In a recent study, low- $k$ thin films with low dielectric constant $(\leq 2.1)$ and high Young's modulus ( $>5$ GPa) were obtained by introducing a remote plasma step between the traditional plasma enhanced chemical vapour deposition and UV curing. This study shows that the UV curing step with a narrow band lamp with wavelength of $172 \mathrm{~nm}$ induced more network $\mathrm{Si}-\mathrm{O}$ and $\mathrm{Si}-\mathrm{H}$ bonds and more densification than the curing step with a broadband lamp with wavelengths higher than $200 \mathrm{~nm}$. As a consequence, the dielectric constant of the narrow band cured film was slightly higher, but Young's modulus and hardness were much improved. Electrical characterization showed good breakdown voltages and a more than sufficient time dependent dielectric breakdown reliability. The broadband lamp was then used to form thicker films which retained very well the characteristics of the thin films. (C) 2011 The Japan Society of Applied Physics

\section{Introduction}

In order to obtain an improved performance of integrated circuits, it is necessary to decrease the delay times of signals in the interconnects. The introduction of copper, in place of aluminium, was a first step, a second is the introduction of insulators with a low dielectric constant. Finding a good low- $k$ material has proven to be more difficult than predicted. The optimization of a low- $k$ material includes finding a compromise between an as low dielectric constant as possible and an as high Young's modulus as possible as well as the feasibility to integrate it into single and/or double damascene structures. ${ }^{1)}$

Recently, spin-on materials have shown to be possible candidates for good low- $k$ films. ${ }^{2)}$ However, in general, industry does not accept spin-on materials for commercial products. Most plasma enhanced chemical vapour deposition (PECVD) based films need two process steps: firstly a PECVD process with co-deposition of a skeleton material and a porogen and secondly a cure, in general using UVlight. ${ }^{1-3)}$ The influence of the UV cure has already been investigated by several authors. ${ }^{1-6)}$ A complete removal of the porogens seemed to be very difficult and the remaining porogens created problems e.g. of high leakage current. ${ }^{4)}$

With this retrospective, the following manufacturing sequence was developed: firstly PECVD deposit a skeleton + porogen $\mathrm{SiCOH}$ material, secondly apply a remote $\mathrm{He} / \mathrm{H}_{2}$ plasma to remove the porogen and finish with a UV cure to strengthen the film. ${ }^{7)}$ A limitation of this method is the reduced depth of penetration of $\mathrm{H}$ radicals to approximately $130 \mathrm{~nm}$, because of their recombination with the pores.

In this paper, the base three-step sequence was further investigated and optimized by applying different cures. Besides, the reproducibility was investigated for films of different thicknesses.

\section{Experimental Methods}

On top of $300 \mathrm{~mm} \mathrm{Si}$ wafers, a $1 \mathrm{~nm}$ thick layer of dry thermal oxide was grown on the $\mathrm{Si}$ wafers prior to the low- $k$

\footnotetext{
*E-mail address: verdonck@imec.be

†Present address: Department of Physics, University of Leuven, Celestijnenlaan 200D, B-3001 Leuven, Belgium.
}

deposition in order to obtain better quality capacitancevoltage $(C-V)$ curves. Then, the advanced low- $k$ (ALK) films were deposited and processed (a sequence of PECVD deposition, remote plasma and UV cure) and characterized as described in detail in ref. 7. In this work we studied the influence of two types of UV cures: two different types of lamps were evaluated: a narrow band lamp with $172 \mathrm{~nm}$ light (NB) and a broad band lamp with wavelengths higher than $200 \mathrm{~nm}(\mathrm{BB})$.

Films of final thicknesses of up to $120 \mathrm{~nm}$ were deposited in one sequence. Films of $200 \mathrm{~nm}$ thickness were obtained either by applying twice the sequence for obtaining the $100 \mathrm{~nm}$ thick films (with BB cure) or by applying twice the deposition and plasma sequence, followed by one double as long BB cure.

Thicknesses of the films were measured by spectroscopic ellipsometry and by X-ray reflection (XRR). Mass was measured with a high resolution, $300 \mathrm{~mm}$ wafer compatible, in-line mass measurement system with an accuracy better than $0.1 \mathrm{mg}$.

Fourier transform infra-red (FTIR) measurements were performed after each subsequent process step for the ALK films with a resolution better than $1 \mathrm{~cm}^{-1}$, averaging 64 spectra within the $400-4000 \mathrm{~cm}^{-1}$ range. For every FTIR analysis, the background spectrum and the substrate spectrum (silicon and $1 \mathrm{~nm}$ of thermal oxide) were subtracted.

The surface of the low- $k$ films was characterized by water contact angle (WCA), taking the average of five measurements.

The Young's modulus of the low- $k$ dielectric films was measured by the nano-indentation technique using a Nanoindenter $\mathrm{XP}^{\circledR}$ system with a dynamic contact module (DCM) and a continuous stiffness measurement (CSM) option under the constant strain rate condition $\left(0.05 \mathrm{~s}^{-1}\right)$. A standard three-sided pyramid diamond indenter tip (Berkovich) was used for the indentation experiments. In order to minimize the effect of the underlying substrate and improve the reliability of the extracted data, different key factors were taken into account. ${ }^{8)}$ In this way, a relative comparison of Young's modulus for the two investigated UV curing conditions was still valid despite the rather low film thickness. This is nicely illustrated by comparing the 
results for 100 and $200 \mathrm{~nm}$ ALK BB cured films, which reveal comparable Young's modulus values (see the results shown in Table IV).

Beside the Young's modulus, the adhesion of the low- $k$ material to the liner is also an important characteristic. The interface adhesion strength is commonly characterized by the critical strain energy release rate, $G_{c}$. The four-point bending test is a powerful, commonly used technique to quantify the interface adhesion strength. ${ }^{9-11)}$ The following procedure was followed. The investigated low- $k$ films were first deposited on $300 \mathrm{~mm} \mathrm{Si}$ wafers, plasma treated and UV cured. The process of record (POR) liner ( $\mathrm{SiCN}$ based) was deposited on top of these films, followed by a $1 \mu \mathrm{m}$ thick $\mathrm{SiO}_{2}$ PECVD film, which serves as a buffer layer during the four-point bending tests. To produce specimens for four-point bending tests, the film stack is then sandwiched in between silicon substrates using an epoxy (EPO-TEK 353ND) and cured at $125^{\circ} \mathrm{C}$ for $120 \mathrm{~min}$. Once cured, the samples were diced into rectangular pieces of sizes $60 \times 5 \mathrm{~mm}^{2}$. The individual four-point bending samples were centrally notched at $75 \%$ of the total silicon thickness. Two different sample configurations were tested, with the notch prepared either at the silicon side, or at the film side. For each investigated film stack, 10 samples were tested using a high precision, micro-mechanical test system using a loading rate of $0.5 \mu \mathrm{m} / \mathrm{s}$. During four-point bending, a pre-crack initiates from the pre-notch, propagates towards the weakest interface in the thin-film stack and then grows stably along the weakest interface. X-ray photoelectron spectroscopy (XPS) was used to reveal the exact fracture location (at a certain interface or within which film).

$C-V$ measurements at $100 \mathrm{kHz}$ were performed for $k$-value extractions by using an impedance analyzer (HP4284A precision LCR meter). Metal-insulator-semiconductor (MIS) planar capacitors were formed after e-beam evaporation of Pt dots upon the blanket dielectric films through shadow masks. For each sample, different dot sizes were measured in order to verify that the capacitance scaled with dot area and to estimate the measurement error which is typically better than $2 \%$. Finally, the dissipation factor was always monitored so as to make sure that it was always lower than 0.1.12) Taking into consideration the accuracy from the thickness measurements and the obtained capacitance values, the final error on the $k$-value was estimated to be \pm 0.1 .

More electrical measurements were conducted using a special test structure based on MIS planar capacitors, designed to investigate the intrinsic electrical properties of thin films. ${ }^{13,14)}$ Voltage ramp measurements were performed at $25^{\circ} \mathrm{C}$, on capacitors with an area ranging from $20 \times$ $20 \mu \mathrm{m}^{2}$ up to $1 \times 1 \mathrm{~mm}^{2}$. The thicknesses used in the calculations of the breakdown fields were measured by ellipsometry in the actual structures used for the electrical measurements. Time-dependent dielectric breakdown (TDDB) measurements were performed at $100^{\circ} \mathrm{C}$ on capacitors of $100 \times 100 \mu \mathrm{m}^{2}$ area. At least 4 different electric field strengths were applied, for each of which at least 10 structures were measured. All measurements were done at approximately the same location on the wafer for all films, to exclude any effects originating from within wafer
Table I. Mass removed by the different treatments, final thicknesses, open porosity, dielectric constant and water contact angle for different ALK films.

\begin{tabular}{ccccccc}
\hline $\begin{array}{c}\text { Nominal } \\
\text { film }\end{array}$ & $\begin{array}{c}\text { Mass } \\
\text { removed } \\
\text { by plasma } \\
(\%)\end{array}$ & $\begin{array}{c}\text { Mass } \\
\text { removed } \\
\text { by cure } \\
(\%)\end{array}$ & $\begin{array}{c}\text { Final } \\
\text { thickness } \\
(\mathrm{nm})\end{array}$ & $\begin{array}{c}\text { Open } \\
\text { porosity } \\
(\%)\end{array}$ & $k$ & $\begin{array}{c}\text { WCA } \\
(\mathrm{deg})\end{array}$ \\
\hline $\begin{array}{c}60 \mathrm{~nm} \mathrm{NB} \\
\text { cure }\end{array}$ & 39.1 & <exp. error & 59 & 45 & 2.2 & 86 \\
$\begin{array}{c}60 \mathrm{~nm} \mathrm{BB} \\
\text { cure }\end{array}$ & 38.4 & <exp. error & 63 & 45 & 2.1 & 88 \\
$\begin{array}{c}90 \mathrm{~nm} \mathrm{NB} \\
\text { cure }\end{array}$ & 30.0 & 4.7 & 91 & 43 & 2.1 & 88 \\
$\begin{array}{c}100 \mathrm{~nm} \mathrm{BB} \\
\text { cure }\end{array}$ & 30.0 & 3.0 & 104 & 44 & 2.1 & 91 \\
$\begin{array}{c}2 \mathrm{nd} \times 100 \\
\text { nm }\end{array}$ & 29.7 & 4.3 & 200 & 42 & 2.1 & 91 \\
$\begin{array}{c}200 \mathrm{~nm}, \\
1 \mathrm{cure}\end{array}$ & 30.7 & 6.9 & 201 & 42 & 2.0 & 91 \\
\hline
\end{tabular}

non-uniformities. For the Weibull fit of the TDDB distributions, a maximum likelihood estimation method was used. To extrapolate the empirical results to user conditions, the E-model was used.

\section{Results and Discussion}

Table I shows the influence of the different UV cures on different characteristics of the different investigated films. This table shows that by far most of the mass removal, which indicates the removal of the porogens, was caused by the remote plasma. The extra UV cures removed much less porogen - because there was much less porogen present in the films after the remote plasma. Narrow band UV cures only removed approximately $80 \%$ of the mass removed by the remote plasmas: the remaining porogen content in the films after UV cure only was much higher as can be seen when comparing the UV ellipsometric spectra, as shown in refs. 4 and 7.

The mass removal data already show that the NB lamp cure influenced the film more than the BB lamp cure: more mass was removed but the film thickness was reduced even more by the NB cure: some densification took place. On the other hand, the open porosity remained the same within the experimental error for all the samples. The differences in dielectric constant were never higher than 0.1 , as long as the Pt evaporation and $C-V$ measurements were done within 48 hs after the UV cure. When the $C-V$ measurements were done after more than one week, the $k$-value for the $60 \mathrm{~nm}$ NB sample increased to 2.4 , probably due to water absorption, but a thermal treatment of $20 \mathrm{~min}$ at $300^{\circ} \mathrm{C}$ reduced it back to 2.2. Although the differences between samples with $\mathrm{NB}$ and $\mathrm{BB}$ cure are small, the $k$ value is systematically somewhat higher for the films cured with the NB lamp.

The water contact angle also was very similar, although the systematically lower value for the NB cure indicated a somewhat less hydrophobic film than for the BB cure.

FTIR analyses, however, showed significant differences in the composition of the film, as shown in Fig. 1 and Table II. $\mathrm{BB}$ exposure did not significantly decrease the $\mathrm{Si}-\mathrm{CH}_{3}$ peak around $1280 \mathrm{~cm}^{-1}$ nor the $\mathrm{CH}_{3}$ peak around $2800 \mathrm{~cm}^{-1}$ (Fig. 1, inset a), while NB exposure decreased both by 
Table II. Relative change of FTIR peak heights (as deposited films $=100 \%$ ) for the nominally $90 \mathrm{~nm}$ NB ALK and $100 \mathrm{~nm}$ BB ALK films.

\begin{tabular}{lccc}
\hline & $\mathrm{CH}_{3}$ & $\mathrm{Si}-\mathrm{CH}_{3}$ & $\mathrm{Si}-\mathrm{H}$ \\
$(\%)$ & $(\%)$ & 0 \\
\hline After remote plasma & -50 & -9 & 0 \\
After extra NB cure & -75 & -36 & +400 \\
After extra BB cure & -50 & -9 & 0 \\
\hline
\end{tabular}

Table III. Mechanical properties, Young's modulus, and hardness of different ALK films.

\begin{tabular}{lcc}
\hline \multicolumn{1}{c}{ Film } & $\begin{array}{c}\text { Young's } \\
\text { modulus } \\
(\mathrm{GPa})\end{array}$ & $\begin{array}{c}\text { Hardness } \\
(\mathrm{GPa})\end{array}$ \\
\hline $90 \mathrm{~nm}$ ALK, NB cure & $5.9 \pm 0.7$ & $0.59 \pm 0.07$ \\
$100 \mathrm{~nm}$ ALK, BB cure & $3.5 \pm 0.4$ & $0.36 \pm 0.04$ \\
$2 \times 100 \mathrm{~nm}$ ALK, 2 BB cure & $3.2 \pm 0.3$ & $0.35 \pm 0.05$ \\
$2 \times 100 \mathrm{~nm}$ ALK, 1 BB cure & $3.3 \pm 0.4$ & $0.37 \pm 0.06$ \\
\hline
\end{tabular}

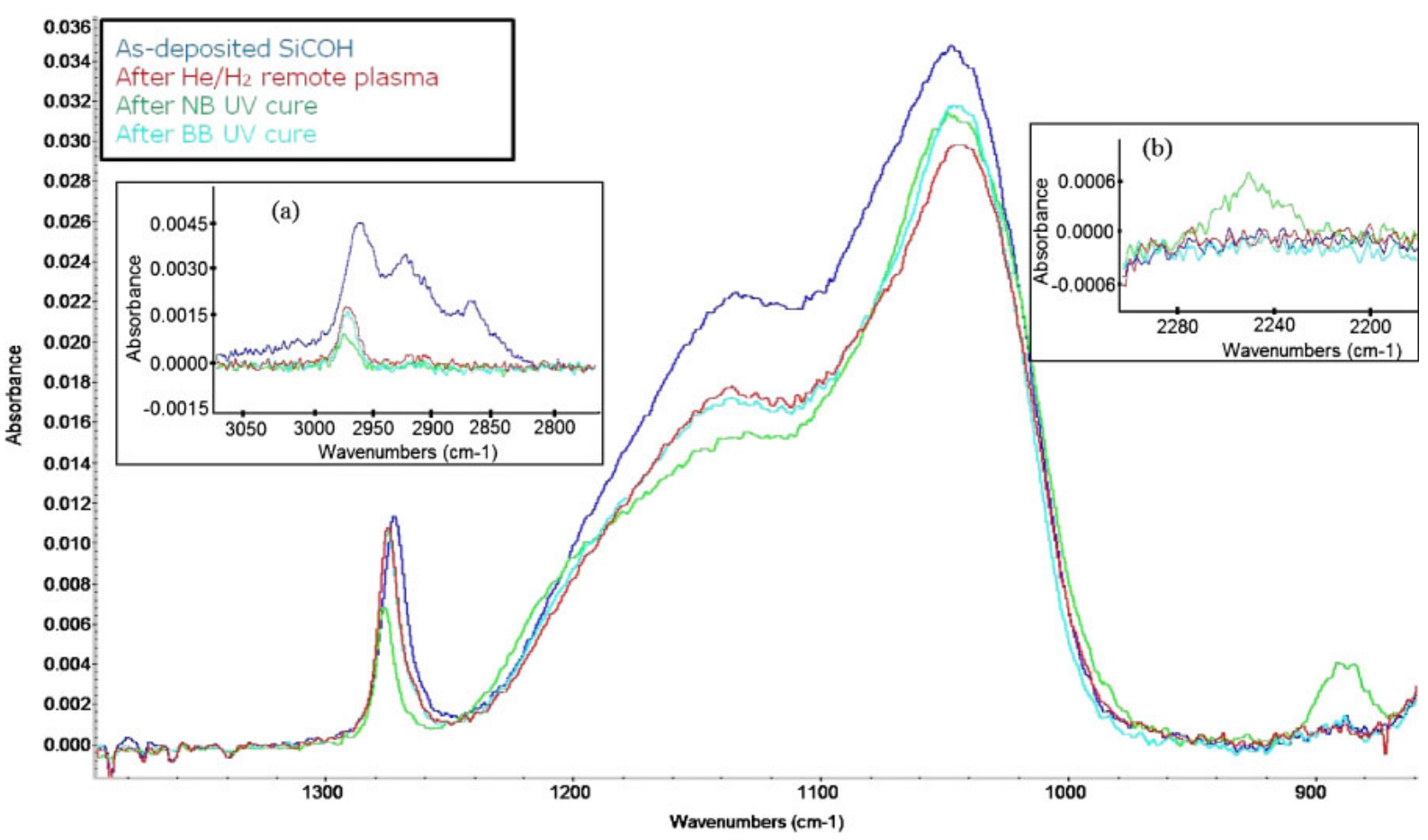

Fig. 1. (Color online) FTIR spectra of nominally 90 and $100 \mathrm{~nm}$ ALK films, as deposited, after remote plasma and after extra NB or BB cure.

respectively $36 \%$ and $50 \%$. Again, this is a clear indication that the NB cure removes $\mathrm{CH}$ based hydrophobic groups. It is also very important to observe that the remote plasma and the BB cure decreased a lot the peaks in the 2800$3000 \mathrm{~cm}^{-1}$ range, but only lightly affected the $\mathrm{Si}-\mathrm{CH}_{3}$ peak around $1280 \mathrm{~cm}^{-1}$. Hence the non-bound $\mathrm{CH}_{x}$ molecules disappeared and pores were formed. On the other hand, the $\mathrm{Si}-\mathrm{CH}_{3}$ molecules remained in the film, which retained its hydrophobic characteristics.

The $\mathrm{Si}-\mathrm{H}$ peak around $2250 \mathrm{~cm}^{-1}$ was only visible after the NB exposure (Fig. 1, inset b) while the $\mathrm{Si}-\mathrm{H}$ peak around $890 \mathrm{~cm}^{-1}$ increased by a factor of 5 after the NB exposure. These results can be explained by the fact that the $172 \mathrm{~nm}$ photon is energetic enough to break the $\mathrm{Si}-\mathrm{C}$ band while a $200 \mathrm{~nm}$ photon is not, as explained in more detail in ref. 5 .

The FTIR spectrum in the $1000-1250 \mathrm{~cm}^{-1}$ range also shows more network $\mathrm{Si}-\mathrm{O}$ for the NB cured film than for the other treated films, as the peak for the convolution of the network and the suboxide peaks moves to a somewhat higher wave number. There was clearly less cage type of Si-O bonds in the NB cured films, as can be seen in the much lower shoulder around $1140 \mathrm{~cm}^{-1}{ }^{15)}$ The network Si-O bond is the strongest bond and the cage $\mathrm{Si}-\mathrm{O}$ bond is the weakest, hence from this spectrum one would expect better mechanical characteristics for the NB cured film than for the BB cured film. This is confirmed below and in Table III, where the nanoindentation results are presented.

Because the dielectric constant of the films with the BB cure was consistently lower, different, higher thicknesses were investigated with the $\mathrm{BB}$ cure.

When comparing the characteristics for the different thicknesses, it is clear that the remote plasma removed relatively more mass (probably porogen) from the thinner $60 \mathrm{~nm}$ film than from the $90 \mathrm{~nm}$ film (a standard NB UV cure immediately after deposition removes approximately $24 \%$ of the mass). Both UV cures were able to remove (at least part of) the still remaining porogen. UV spectroscopic ellipsometry indicated the presence of little or no porogen in all ALK films after the plasma + cure treatments.

As shown in our previous study, ${ }^{7)}$ it was difficult for the remote plasma to remove the porogens efficiently from films thicker than $130 \mathrm{~nm}$. For practical integration schemes, certainly for double damascene integration, it is necessary to obtain films thicker than $130 \mathrm{~nm}$. As said in the experimental section, these thicker films were obtained by repeating the deposition-plasma sequence, followed by 1 cure after each plasma or by a longer cure after the second plasma. Table I 
shows results of mass removal for these process steps. The mass removed by the first plasma and the first BB cure are shown in the fourth row, for the $100 \mathrm{~nm}$ thick film. The fifth row shows how much mass the second plasma treatment and the second cure removed: The mass removed by the remote plasma of the second $100 \mathrm{~nm}$ was, within the reproducibility, the same of the first $100 \mathrm{~nm}$ film, no extra porogens were hence removed from the lowest $100 \mathrm{~nm}$ film with the second plasma. However, the BB cure was able to remove some more mass, also from the lowest part of the final $200 \mathrm{~nm}$ film. The sixth row shows the result of the mass removed by the second plasma, but for this sample, no UV cure had yet taken place at that moment. This second plasma apparently is able to remove a small portion of the porogen still remaining in the lowest $100 \mathrm{~nm}$ of the film. On the other hand, the longer $\mathrm{BB}$ cure is certainly able to remove also porogens from the lower $100 \mathrm{~nm}$ of the film. This shows, again, that the UV cure was able to modify the material deeper than the remote plasma does. In the end, one may say that the longer, one step $\mathrm{BB}$ cure is as efficient as the two short BB cures.

The characteristics of the obtained $200 \mathrm{~nm}$ films are also shown in Table I. The $200 \mathrm{~nm}$ thick film with only one long cure had the lowest dielectric constant, probably because the second $\mathrm{He}-\mathrm{H}_{2}$ plasma was able to remove some extra porogen from the lowest $100 \mathrm{~nm}$ of the film.

The mechanical characteristics of the different investigated films are shown in Table III. The nanoindentation showed that the films cured with the narrow band, short wavelength lamp acquired much better mechanical characteristics, both Young's modulus and hardness. The small differences in measured Young's modulus between the samples with the BB cure, for the 100 and $200 \mathrm{~nm}$ thick films, were probably due to the difference in film thickness, causing a different substrate effect. The fact that this measured difference was very small, is a strong indication that the accuracy is quite good and that the comparison between the results of the different films is valid. ${ }^{7)}$ To the authors' best knowledge, the combination of a 2.1-2.2 dielectric constant with a 5.9 Young's modulus is the best combination ever reported for PECVD deposited films and also favourable to most spin-on-glass (SOG) type low- $k$ films. This better mechanical characteristic is completely compatible with the fact that much less cage and more network $\mathrm{Si}-\mathrm{O}$ bonds were found in the FTIR spectrum of the NB cured film, as explained above.

Figure 2 shows the combination of Young's modulus and dielectric constant for different CVD and spin-on low- $k$ materials. All the values in this figure were obtained by very similar measurement procedures for all these materials, hence it is possible to make a significant comparison. It shows the very good combination of electrical and mechanical characteristics of the films studied in this paper.

The results in Table I also show that one may choose the type of cure as a function of the application. If the lowest dielectric constant is the final goal, then the BB cure is preferred. If the combination of high hardness and Young's modulus with a slightly higher $k$-value is preferred, then the NB cure is more adequate.

The four point bending measurements were not able to yield exact values of the adhesion strength between the low-

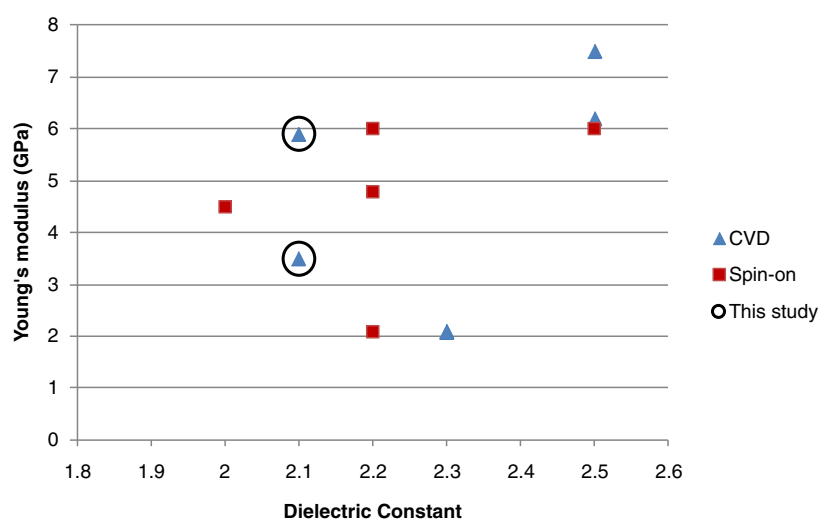

Fig. 2. (Color online) Young's modulus versus dielectric constant for different PECVD and spin-on materials.

Table IV. Breakdown voltage and reliability properties as determined by TDDB testing.

\begin{tabular}{lcccccc}
\hline & $\begin{array}{c}V_{\mathrm{BD}} \\
(\mathrm{MV} / \mathrm{cm})\end{array}$ & $\begin{array}{c}\gamma \\
(\mathrm{cm} / \mathrm{MV})\end{array}$ & $\beta$ & $\begin{array}{c}t_{63 \%}(\text { years }) \\
\text { at } \\
E=1 \mathrm{MV} / \mathrm{cm}\end{array}$ & $\begin{array}{c}E(\mathrm{MV} / \mathrm{cm}) \\
\text { at } t_{63 \%}=10 \\
\text { years }\end{array}$ & $\begin{array}{c}E(\mathrm{MV} / \mathrm{cm}) \\
\text { at } t_{0.01 \%}=10 \\
\text { years }\end{array}$ \\
\hline $\begin{array}{l}\text { ALK NB, } \\
60 \mathrm{~nm}\end{array}$ & 6.9 & -4.55 & 2.11 & 47 & 1.34 & 0.38 \\
$\begin{array}{l}\mathrm{ALK} \mathrm{NB}, \\
120 \mathrm{~nm}\end{array}$ & 7.6 & -3.51 & 5.17 & 90 & 1.63 & 1.12 \\
$\begin{array}{l}\mathrm{ALK} \mathrm{BB}, \\
120 \mathrm{~nm}\end{array}$ & 7.3 & -3.72 & 5.07 & 133 & 1.70 & 1.21 \\
\hline
\end{tabular}

$k$ films and the liner, because the cleavage always occurred between the liner and the $\mathrm{SiO}_{2}$ crack propagation film. The only conclusion we can draw is that the critical strain energy release rate between the different ALK's and the liner is better than $2.8 \mathrm{~J} / \mathrm{m}^{2}$ and better than the adhesion between liner and $\mathrm{SiO}_{2}$, hence satisfactory.

Beside the dielectric constant, other important electrical characteristics are the breakdown voltage and TDDB. The breakdown voltage and the TDDB were measured using MIS planar capacitors, described in the experimental section and in more detail in refs. 13 and 14 . The results for the breakdown voltage (at $25^{\circ} \mathrm{C}$ ) and TDDB measurements (at $100^{\circ} \mathrm{C}$ ) can be found in Table IV. The breakdown voltages are relatively high for such a low- $k$ material, similar to breakdown voltages of materials with a dielectric constant of 2.3. The TDDB results are very good: the lifetime exceeds 10 years for all investigated films. The factor indicating the spread $(\beta)$ is excellent, while the shape factor $(\gamma)$ is similar to other PECVD low- $k$ films with dielectric constants of 2.3 and $2.5{ }^{16)}$

Figure 3 shows a plot of lifetime $\left(t_{63 \%}\right)$ versus electric field, from TDDB measurements. No significant difference is found between ALK NB and ALK BB. At the experimental conditions, lifetimes are lower for $60 \mathrm{~nm}$ than for $120 \mathrm{~nm}$, but at user conditions the values become similar. The material clearly meets the 10 years specification at $1 \mathrm{MV} / \mathrm{cm}$ and is thus intrinsically reliable. This is true even for the used extrapolation according to the E-model, for which it has recently been shown that it is actually much too conservative when $\mathrm{Cu}$ is present in the dielectric film. ${ }^{17,18)}$ 


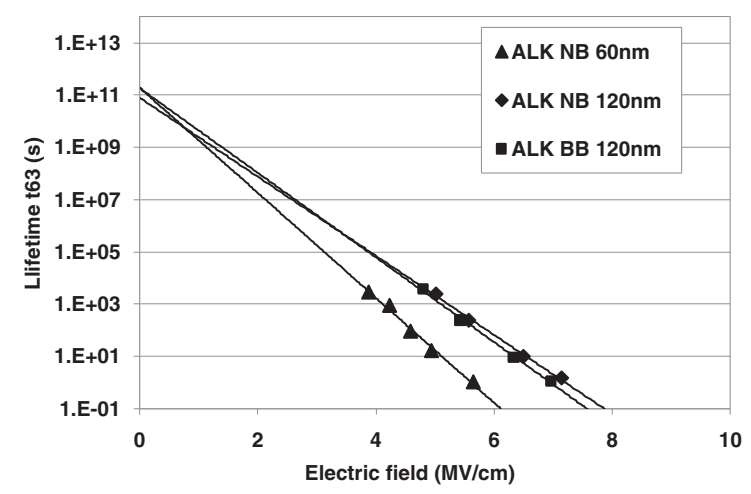

Fig. 3. TDDB results and extrapolations following the E-law for different ALK films, all showing results better than 10 years lifetime.

\section{Conclusions}

A relatively new procedure to manufacture low- $k$ films was evaluated quite exhaustively. In order to obtain a dielectric constant of 2.1 or less, starting from a PECVD film, a remote plasma step was used to remove more than $90 \%$ of the porogens. The influence of subsequent UV cures, either with a narrow band lamp with a wavelength of $172 \mathrm{~nm}$ or a broad band lamp with wavelengths higher than $200 \mathrm{~nm}$, was studied. The narrow band lamp modified the low- $k$ film substantially, inducing more network type $\mathrm{Si}-\mathrm{O}$ bonds, but also more $\mathrm{Si}-\mathrm{H}$ bonds; besides, the film was somewhat densified. As a results the dielectric constant increased somewhat, but also the hardness and Young's modulus increased significantly, obtaining a film with $k=2.1$ and $E=5.9 \mathrm{GPa}$. Almost no skeleton modification is observed when the broad band UV cure was used, hence lower dielectric constants, combined with lower hardness and Young's modulus were obtained. For the investigated films, the adhesion to the liner was satisfactory. Breakdown voltages and TDDB measurements showed similar or even better results than for $k=2.3$ and 2.5 materials.

\section{Acknowledgements}

The authors would like to thank Leo Farrell, Ivan Ciofi, Kristof Croes, Zsolt Tökei, and Larry Zhao for helpful discussions.

1) K. Maex, M. R. Baklanov, D. Shamiryan, F. Iacopi, S. Brongersma, and Z. Sh. Yanovitskaya: J. Appl. Phys. 93 (2003) 8793.

2) W. Volksen, R. Miller, and G. Dubois: Chem. Rev. 110 (2010) 56.

3) A. Grill, V. Patel, K. P. Rodbell, E. Huang, M. R. Baklanov, K. P. Mogilnikov, M. Toney, and H.-C. Kim: J. Appl. Phys. 94 (2003) 3427.

4) P. Marsik, P. Verdonck, D. de Roest, and M. R. Baklanov: Thin Solid Films 518 (2010) 4266

5) L. Prager, P. Marsik, L. Wennrich, M. R. Baklanov, S. Naumov, L. Pistol, D. Schneider, J. W. Gerlach, P. Verdonck, and M. R. Buchmeiser: Microelectron. Eng. 85 (2008) 2094.

6) P. Verdonck, D. De Roest, S. Kaneko, R. Caluwaerts, N. Tsuji, K. Matsushita, N. Kemeling, Y. Travaly, H. Sprey, M. Schaekers, and G. Beyer: Surf. Coat. Technol. 201 (2007) 9264.

7) A. M. Urbanowicz, K. Vanstreels, P. Verdonck, D. Shamiryan, S. De Gendt, and M. R. Baklanov: J. Appl. Phys. 107 (2010) 104122.

8) K. Vanstreels and A. M. Urbanowicz: J. Vac. Sci. Technol. B 28 (2010) 173.

9) R. H. Dauskardt, M. Lane, Q. Ma, and N. Krishna: Eng. Fract. Mech. 61 (1998) 141.

10) Q. Ma, H. Fujimoto, P. Flinn, V. Jain, F. Adibi-Rizi, F. Moghadam, and R. H. Dauskhardt: Proc. MRS Spring Meet., San Fransisco, Materials Reliability in Microelectronics V, 1995, p. 91.

11) Q. Ma: J. Mater. Res. 12 (1997) 840.

12) I. Ciofi, M. R. Baklanov, Z. Tökei, and G. P. Beyer: Microelectron. Eng. 87 (2010) 2391.

13) L. Zhao, Z. Tökei, G. Giai Gischia, H. Volders, and G. Beyer: Proc. IEEE Int. Interconnect Technology Conf., 2009, p. 206.

14) L. Zhao, Z. Tökei, G. Giai Gischia, M. Pantouvaki, K. Croes, and G. Beyer: Proc. IEEE Int. Reliability Physics Symp., 2009, p. 848.

15) A. Grill and D. A. Neumayer: J. Appl. Phys. 94 (2003) 669.

16) E. Van Besien, L. Zhao, D. De Roest, M. Pantouvaki, M. Baklanov, P. Verdonck, Z. Tökei, and G. Beyer: to be published in Microelectron. Eng. and published in Proc. AMC 2010, 2010, Abstract number 43.

17) K. Croes, G. Cannatá, L. Zhao, and Z. Tökei: Microelectron. Reliab. 48 (2008) 1384.

18) K. Croes and Z. Tökei: Proc. IEEE Int. Reliability Physics Symp., 2010, p. 549 . 